\title{
CMMSE: Combinatorial structures and finite-dimensional alternative algebras
}

\author{
Manuel Ceballos ${ }^{1}$ \\ ${ }^{1}$ University of Seville
}

September 29, 2021

\begin{abstract}
In this paper, the link between combinatorial structures and alternative algebras is studied, determining which configurations are associated with those algebras. Moreover, the isomorphism classes of each 2-dimensional configuration associated with these algebras is analyzed, providing a new method to classify them. In order to complement the theoretical study, two algorithmic methods are implemented: the first one constructs and draws the (pseudo)digraph associated with a given alternative algebra and the second one tests if a given combinatorial structure is associated with some alternative algebra.
\end{abstract}

\section{Hosted file}

C_alternativeCMMSE.pdf available at https://authorea.com/users/438470/articles/539666-cmmsecombinatorial-structures-and-finite-dimensional-alternative-algebras 